\title{
Video Article \\ A Modified Lean and Release Technique to Emphasize Response Inhibition and Action Selection in Reactive Balance
}

\author{
David A.E. Bolton ${ }^{1}$, Mahmoud Mansour ${ }^{2}$ \\ ${ }^{1}$ Department of Kinesiology \& Health Science, Utah State University \\ ${ }^{2}$ Department of Electrical \& Computer Engineering, Utah State University
}

Correspondence to: David A.E. Bolton at dave.bolton@usu.edu

URL: https://www.jove.com/video/60688

DOI: doi:10.3791/60688

Keywords: Behavior, Issue 157, balance, posture, lean \& release, cognition, response inhibition, decision-making, falls

Date Published: 3/19/2020

Citation: Bolton, D.A., Mansour, M. A Modified Lean and Release Technique to Emphasize Response Inhibition and Action Selection in Reactive Balance. J. Vis. Exp. (157), e60688, doi:10.3791/60688 (2020).

\section{Abstract}

Assessment of reactive balance traditionally imposes some type of perturbation to upright stance or gait followed by measurement of the resultant corrective behavior. These measures include muscle responses, limb movements, ground reaction forces, and even direct neurophysiological measures such as electroencephalography. Using this approach, researchers and clinicians can infer some basic principles regarding how the nervous system controls balance to avoid a fall. One limitation with the way in which these assessments are currently used is that they heavily emphasize reflexive actions without any need to revise automatic postural reactions. Such an exclusive focus on these highly stereotypical reactions would fail to adequately address how we can modify these reactions should the need arise (e.g., avoiding an obstacle with a recovery step). This would appear to be a glaring omission when one considers the enormous complexity of the environments we face daily. Overall, the status quo when evaluating the neural control of balance fails to truly expose how higher brain resources contribute to preventing falls in complex settings. The present protocol offers a way to require suppression of automatic, but inappropriate corrective balance reactions, and force a selection among alternative action choices to successfully recover balance following postural perturbation.

\section{Video Link}

The video component of this article can be found at https://www.jove.com/video/60688/

Introduction

Despite the recognized correlation between falls and cognitive decline ${ }^{1,2,3}$, a major gap persists in understanding what the brain actually does to help us avoid a fall. In theory, cognitive demands would be accentuated as environmental complexity increases and in situations where we need to revise instinctive behavior. However, most balance tests fail to effectively tax higher brain function, instead emphasizing reflexive righting reactions. While factors such as response speed are essential to prevent a fall, additional cognitive factors, such as inhibitory control and/or the ability to select appropriate action based on a given context may also be important in certain situations. As a result, one reason we may fail to understand the brain's role in reactive balance is due to research protocols currently in use. Rogers et al. recently summarized the different ways in which balance control has been assessed using external perturbation ${ }^{4}$. These methods include platform translation, tilts and/or drops, as well as the use of automated systems that push, pull, or remove postural support. Despite the large variety of techniques used to disrupt upright equilibrium, the ensuing corrective reactions are almost always made in an unobstructed environment, thus minimizing constraints on movement. Here, we propose a method where cognitive processes are required to override prepotent action and select suitable responses among alternatives in a reactive balance task.

A common way to test reactive balance is to impose relatively small postural perturbations that can be countered using a fixed support (typically feet-in-place) reaction ${ }^{5,6,7,8,9}$. Comparatively fewer studies have focused on change-of-support balance reactions in response to perturbations via waist pulls, platform translation, and release from a support cable As an example, see Mansfield et al. ${ }^{10}$. The importance of the latter group can be appreciated by recognizing that when perturbations are large, change-of-support reactions are the only option to recover stability ${ }^{11}$. In fact, even for smaller perturbations that could be managed using feet-in-place (i.e., hip and/or ankle) strategies, people frequently prefer to step when given the choice ${ }^{11}$. The value in studying such change-of-support reactions lies not only in the fact that a greater magnitude of perturbation must be countered, but also the challenges that emerge when repositioning the limbs to establish a new support base. The presence of affordances and/or constraints on action are a regular part of many real-world settings. This forces a selection process to establish a new base of support when a loss of balance occurs. To adapt behavior to complex environments, there is a heightened demand on higher brain resources. This is especially true when the limbs must establish a new base of support. To emphasize and expose cognitive roles in reactive balance the need to reintroduce clutter and force a change-of-support strategy with the limbs seems logical

One simple way to deliver an externally induced postural perturbation is the lean \& release technique, where an individual is suddenly released from a supported forward lean. This approach allows assessment of compensatory reactions to avoid a forward fall and has been successfully used in both healthy and clinical populations ${ }^{12,13,14}$. Although the lean \& release technique is somewhat basic, it offers valuable insight into reactive balance capacity (e.g., how quickly someone can initiate a recovery step, or to determine the number of steps required to regain 
stability). For present purposes, the lean \& release technique provides a simple way to explore cognitive roles in reactive balance because many of the perturbation characteristics are held constant. This provides greater experimental control over variables specifically relevant to action selection and response inhibition. While other modes of postural perturbation typically rely on unpredictability in terms of perturbation direction, amplitude, and timing, the surrounding environment is always constant. Even in studies where leg blocks have been used to emphasize reachto-grasp reactions ${ }^{15}$ the blocks are fixed in place with no need to quickly adapt stepping behaviors based on the presence or absence of a leg block. With the presently proposed method, we can change the environment in a way that demands behavioral adaptation to avoid a fall.

Beyond laboratory settings that inadequately expose cognitive roles in reactive balance, another major issue is a heavy reliance on external measures such as muscle onsets, ground reaction forces, and video motion capture to infer neural processes. While these measures are valuable, exclusive reliance on such measures fails to provide direct insight into the underlying neural mechanisms that contribute to balance. This problem is compounded when considering that much of what the brain may do to prevent a fall in complex environments likely happens before the fall. Predictive roles in fall prevention have recently been discussed more extensively ${ }^{16}$. Research directions include predicting future instability $^{17}$, building visuospatial maps as we move through our environment ${ }^{18}$, and possibly forming contingencies based upon the environment even without foreknowledge of a fall ${ }^{19}$. Revealing such preparation would be entirely inaccessible without use of direct neurophysiological probes.

The modified lean \& release approach as presently proposed offers a means to overcome some of the existing limitations mentioned. This is done by using a testing scenario where the limbs are required to establish a new base of support in a choice-demanding environment. This approach is augmented by including direct measures of brain activity (e.g., transcranial magnetic stimulation, TMS) both before and after postural perturbation, which can complement external measures of force production and motion capture. This combination of experimental features represents an important innovation in the field to expose how the brain contributes to balance in complex settings where response inhibition and selecting actions among options are called for to prevent a fall. Here we demonstrate a novel method for testing reactive balance in a setting that emphasizes the need for cognitive processes to adapt behavior in order to avoid a fall. The combination of obstacles and affordances for action force the need for response inhibition, targeted action, and response selection among options. Moreover, we demonstrate precise temporal control over visual access, timing of neural probes, changing the response environment, and onset of the postural perturbation.

\section{Protocol}

All procedures received approval from the Institutional Review Board at Utah State University and were conducted in accordance with the Declaration of Helsinki.

\section{Participant screening}

1. Have participants provide written informed consent to procedures prior to testing.

2. For testing with TMS, screen participants prior to testing in order to assess their suitability for TMS using guidelines developed by a group of experts $^{20}$

\section{Data acquisition: electromyography (EMG)}

1. Record EMG using surface electrodes and amplify signals (gain $=1,000$; see Table of Materials).

2. Acquire data and bandpass filter $(10-1,000 \mathrm{~Hz})$ using a data acquisition interface and appropriate software (see Table of Materials). Use this device and software to control the various motors, cable release, and occlusion goggles as described later in the methods.

3. Gently abrade the skin surface and wipe with alcohol over the target muscle locations. Fix the surface EMG electrodes onto the target muscles using two-sided tape, and further secure using prewrap to ensure that the electrodes remain fixed, especially during rapid responses with the arms and legs.

4. Collect EMG data from two intrinsic hand muscles on the right hand (first dorsal interosseus, FDI and opponens pollicus, OP) and ankle dorsiflexors on both legs (tibialis anterior, TA).

NOTE: These particular muscles were selected based on their relevance to a reach-to-grasp action or a forward step, but other muscles could be selected as needed. 


\section{Balance testing equipment}

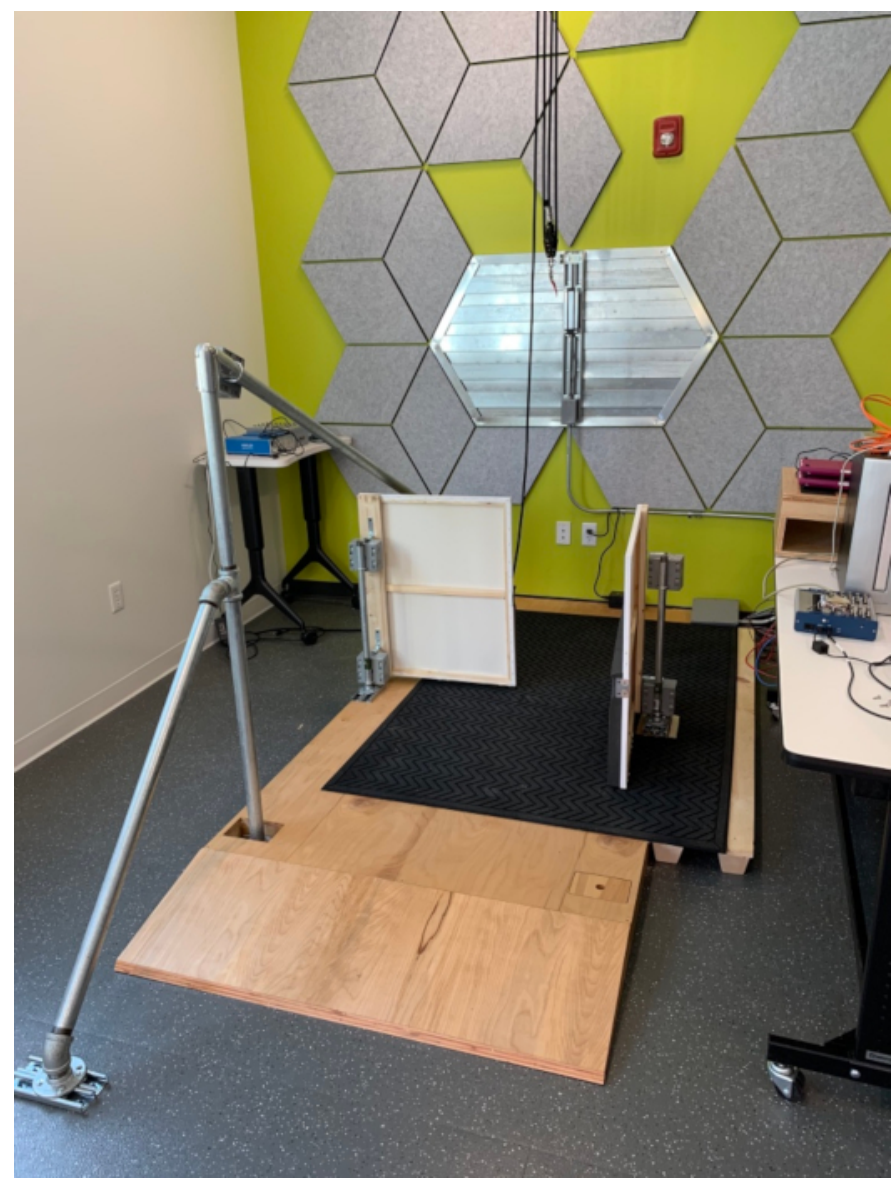

Figure 1. Lean \& release setup with leg blocks. In this example, one leg block is set in the open position, while the other is set to prevent a step. These blocks are moved via computer-controlled motors (grey boxes attached to the support posts). Handle covers are also moved to either block or allow a reach-to-grasp response. Here, the covers are detached to allow full view of the handle. The release magnet is visible on the back wall. All the wiring feeds through the wooden platform itself and enters into the grey circuit box located on the back corner. Please click here to view a larger version of this figure. 


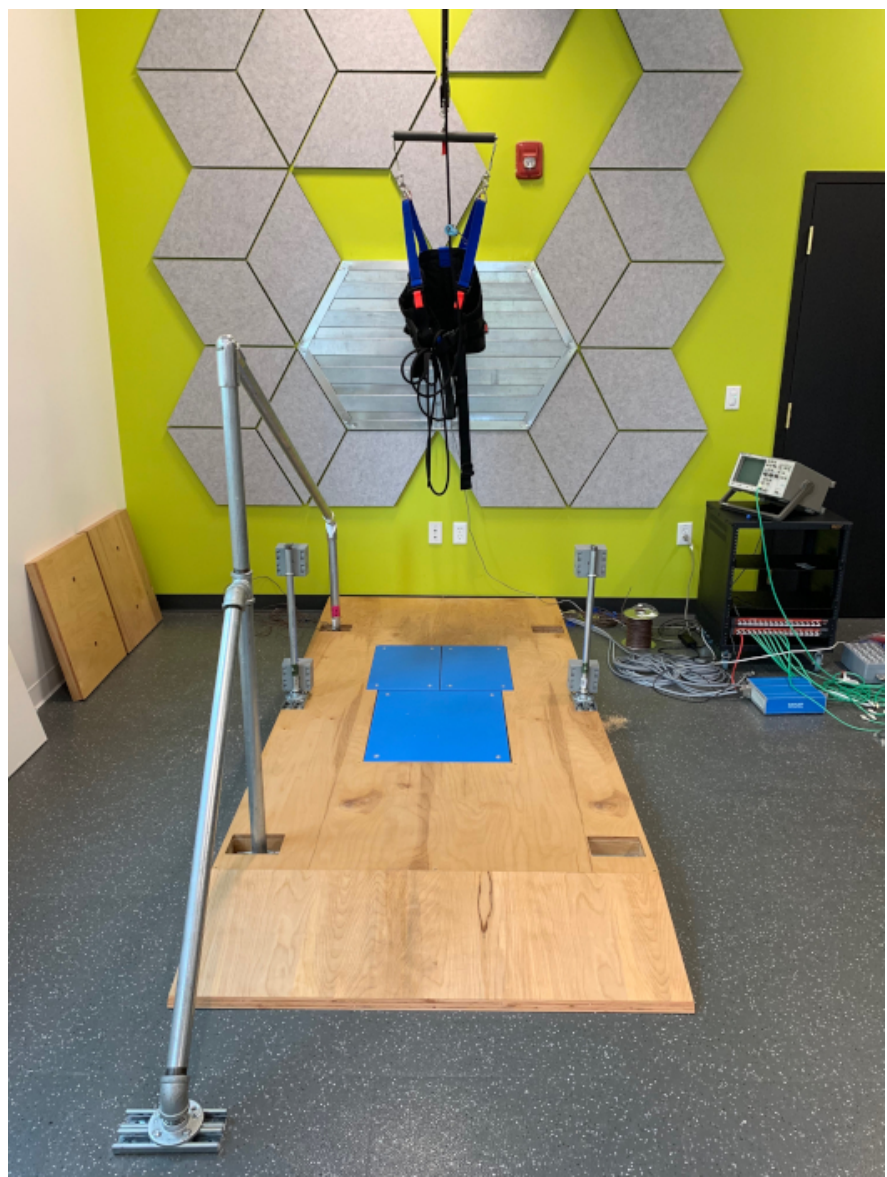

Figure 2. Lean \& release setup with force plates. This figure depicts how three force plates can be optionally embedded into the wooden platform. If force plates are not required, wooden plugs can be set in place. These plugs are visible, leaning on the side wall. This image also shows the safety harness worn by participants. This harness is secured to the ceiling to act as a safety mechanism should the participant fail to recover their balance on their own. Please click here to view a larger version of this figure.

\section{Modified lean \& release system}

1. Use a custom-made, lean \& release cable system to impose forward perturbations (see Figure 1 and Figure 2).

2. Have participants stand in a forward lean position with their feet approximately hip width apart (see Figure 3). Maintain this forward lean using a body harness attached to a cable, which is then secured to the wall behind them. Fasten the cable to the back of the harness (approximately midthoracic level). Fix the support cable to the wall by a magnet. The magnet will be briefly deactivated to release the cable.

3. Make the specific trial procedures (i.e., when the cable is released and the onset of the cable release) unpredictable to the participant Control the precise timing of cable release via computer commands preset into a software configuration. This configuration will allow control of the timing of cable release so that it can be randomized across trials.

NOTE: The software configuration that controls all experimental devices (e.g., triggering the motor to position a leg block) sets the specific trial condition (e.g., if a leg block is present or not). This can be programmed to randomize conditions or deliver them in blocks to control the level of predictability.

4. In addition to this release cable attached to the back of the harness, also secure participants to a support cable hanging from the ceiling. This failsafe cable provides no bodyweight support unless absolutely necessary. If a participant is unable to recover balance on their own, the cable catches them before falling to the ground.

5. Due to the importance of reliable visual information, verify that participants can actually see the handle and leg block when wearing the goggles. Begin each trial by instructing participants to look directly at a fixed point on the floor, about $3 \mathrm{~m}$ in front of them, while holding their head in a comfortable position. Position the participants such that their gaze is set to view the handle in the peripheral visual field and the top portion of the obstacle.

6. Position the body to ensure that the handle is within graspable range. Have the participant lean forward while keeping both feet in contact with the floor. This will require rotation about the ankle while the rest of the body remains in a straight line.

7. Determine the specific lean position as the minimal lean angle where a forward step is necessary to recover balance when the cable is released. This is an iterative process to find a threshold lean angle at the ankle joint, which is the angle where the participant is no longer able to prevent a forward fall using a feet-in-place reaction. Once this is established, verify the lean angle throughout testing using goniometry.

\section{Affordances and constraints on compensatory balance responses}

1. Fix a safety handle onto the wall beside participants on their right side. Use a motorized cover to control access to this handle. If the handle is uncovered, when the participants are released from their supported forward lean it can be used to regain balance. 
2. During trials where the handle is uncovered, place a leg block in front of the participant's legs. The leg block impedes a step, but is not rigidly set in place, meaning that it can be displaced when kicked. Program the leg block to allow free movement and construct it with compliant material to avoid injury.

NOTE: The leg blocks have been constructed to force an 'all-or-none' step decision given that they rise almost 30 inches off the ground (mid-thigh level on most individuals). For researchers interested in a more nuanced blockade of a recovery step, these devices could be modified to use a smaller/shorter obstacle that would then allow an adapted step to clear them.

3. Use a black tarp to cover the handle and block it from view on certain trials. The handle will remain mounted at the same location but will be physically covered to prevent direct visual access and to prevent any supportive grasp. When this support handle is covered, remove the leg block to allow a step reaction if necessary.

\section{Control of vision}

1. Limit vision to the time frame just before postural perturbation and control via liquid crystal goggles (see Table of Materials). When closed, the goggles prevent access to the visual scene so participants are unaware of the forthcoming response condition.

2. Change the specific configuration of the leg block and handle availability for each trial while the goggles are closed so that participants need to quickly perceive the environment once the goggles open. Move the handle cover and the leg block into position via computertriggered, servo motors at the start of each trial. Have the participants wear ear plugs and make motors move continuously during the period of visual occlusion to avoid any advanced cueing for the upcoming condition.

\section{Experimental design}

1. Prior to testing, briefly familiarize participants with how to reach the handle and step forward from a leaning position.

1. Provide participants with full knowledge of the upcoming practice condition and make sure there is no uncertainty. Instruct the participants that once the goggles are open, they will see the handle covered, and the stepping path will be clear. Shortly after, the support cable will release and they will have to step quickly to avoid falling forward.

2. Use similar instructions regarding whether or not the handle is available for grasping to avoiding a step.

3. Throughout testing and practice, instruct the participants to remain relaxed unless prompted to move by a sudden cable release. NOTE: On average, participants require approximately 10 practice attempts before formal testing begins.

2. Randomly change the response setting between trials. If released from the support cable, participants must regain stability by either reaching for the wall-mounted safety handle or stepping forward if the step path is clear.

3. Always close the occlusion goggles at the beginning of each trial, at which time the response setting will be altered. Close the goggles for a randomized period (usually about 3-4 s) to allow the setting to change.

4. When the goggles open, provide one of two possible response settings: (1) the leg block is present and the support handle is present, or (2) no leg block is present and no support handle is present.

NOTE: In the first condition, a support handle is available at a comfortable reach distance and the leg block prevents a step. This setting imposes a context where the only option available is to quickly grasp the available support handle with their right arm. The second condition allows for a recovery step while preventing use of the support handle.

5. On trials where a perturbation does occur, release the cable shortly after the goggles open. This delay period will vary with study requirements, but ranges from $200-1,000 \mathrm{~ms}$.

6. For some trials, do not release to act as a catch trial. This helps avoid anticipatory responses based only on vision.

7. Have each trial last $10 \mathrm{~s}$, with a short pause between trials to allow participants a chance to reset as needed. Give participants a brief rest period in between each test block and allow them to sit. The basic experimental design is depicted in Figure 3 (bottom). NOTE: The total trial number is varied to suit the needs of each study but tends to include approximately 100 trials divided across three to four test blocks. 

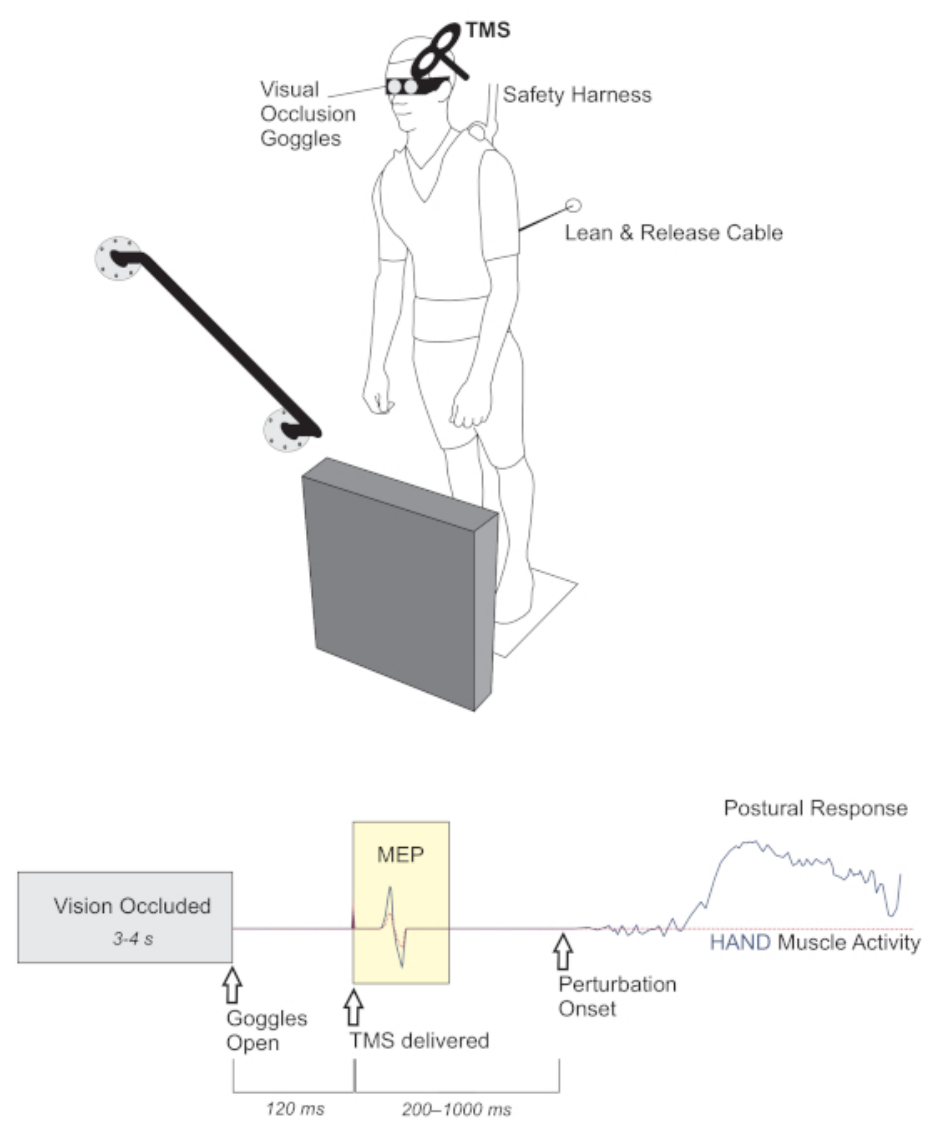

Figure 3. TMS-based method to investigate the impact of perceiving environmental affordances and/or constraints on motor preparation. TOP. A lean \& release apparatus released participants in an unpredictable manner (perturbation test blocks only). The magnitude of perturbation required a rapid change-of-support reaction, using either the arm or leg to re-establish a stable base of support by either reaching to a secure handhold, or taking a forward step. In between trials, vision was occluded using liquid crystal occlusion spectacles and objects in the foreground were rearranged at random. BOTTOM. The timeline depicts when visual access to the environment became available and the timing of TMS probes relative to both visual access and the perturbation. The peak-to-peak amplitude of the muscle response to TMS (i.e., motor evoked potential, MEP) provided an index of corticospinal excitability in the time period before perturbation. This figure presents theoretical response data to demonstrate the hypothesized impact of an affordance for hand action (solid, blue line) versus a trial where the handle is covered (dotted, red line). In this figure, both trials/conditions are overlaid to illustrate the hypothesized effect of preparing motor output to either facilitate or suppress potential action based on a particular environmental context. Adapted from Figure 1 in Bolton et al. ${ }^{21}$. Note that TMS was used to probe corticospinal excitability in this example. However, this is only intended to provide a basic representation of the sequence of events using this modified lean \& release. Please click here to view a larger version of this figure.

\section{TMS protocol (optional)}

1. Deliver single-pulse TMS over the hand motor cortical representation while participants are supported in a forward lean. Deliver TMS pulses shortly after opening the goggles but prior to any movement to investigate how viewing the environment impacts the motor set. See Figure 3 to visualize the sequence of events during a trial, including when TMS is delivered.

2. Set the timing for TMS delivery according to the research question. In the representative results, stimulation varied between $100 \mathrm{~ms}$ and 200 ms post-vision. In addition to the response settings listed above, randomly intersperse 'no-vision' reference trials throughout testing to deliver TMS without opening the goggles. The purpose of this condition is to provide a baseline for any task-related changes in motor activity (e.g., increased arousal).

NOTE: Further details on the specific TMS procedures can be found in Bolton et al. ${ }^{21}$ and Goode et al. ${ }^{22}$.

3. Deliver magnetic stimuli to the primary motor cortex (M1) with the stimulating coil oriented approximately $45^{\circ}$ to the sagittal plane (see Table of Materials). Apply stimuli at the optimal position to obtain a motor evoked potential (MEP) in the FDI muscle on the right hand (i.e., the motor 'hotspot').

4. Once the 'hotspot' is found, determine a test stimulus intensity is determined. For the current research purposes, this is the stimulus intensity where the average MEP is approximately $1-1.5 \mathrm{mV}$ peak-to-peak. Fix the TMS coil on this location and reset the coil position if head motion occurs (e.g., following cable release). Determine the test stimulus intensity while subjects stand in forward lean to account for any postural state influence on corticospinal excitability. 


\section{Representative Results}

All exemplar studies presented were conducted with young women and men between 18-30 years of age. The total sample size for each study was as follows: Example 1 (Rydalch et al. ${ }^{23}$ ) included 12 participants, Example 2 (Bolton et al. ${ }^{21}$ ) included 63 participants, and Example 3 (Goode et al. ${ }^{22}$ ) included 19 participants. The reader should refer to the complete studies for details on methods and analyses.

\section{Example 1}

Blocking a rapid recovery step, particularly when stepping was made automatic by frequent repetition, allowed for assessment of response inhibition in a postural context. Here, we compared the leg muscle response when a forward step was either allowed or obstructed ${ }^{23}$. The muscle response from the stepping leg was compared between trials where the participant should reach versus trials where they should step. This was accomplished by comparing the response magnitude of ankle dorsiflexors (tibialis anterior) during reach-to-handle versus step trials. Specifically, the integrated EMG over a $200 \mathrm{~ms}$ window (i.e., $100 \mathrm{~ms}$ to $300 \mathrm{~ms}$ post-perturbation) was used to calculate a muscle response ratio. A smaller value indicated a greater ability to refrain from stepping as described in detail in Rydalch et al. ${ }^{23}$. By using the magnitude of the muscle response, our intention was to provide a sensitive gauge for a tendency to respond with the leg. In this example, the goal of our study was to determine if response inhibition measured with a seated cognitive test (i.e., stop signal task, SST) correlated with performance on a reactive balance task where suppression of a balance recovery step was required. In the balance task, a total of 256 trials were collected, of which $30 \%$ used a leg block. In Figure 4A, we highlight averaged waveforms of individuals that were on opposite ends of the continuum for suppressing step-related leg activity. The scatterplot in Figure 4B depicts a small, but significant correlation between the ability to suppress a blocked step and response inhibition as measured by the stop-signal reaction time.

When interpreting these results, it is important to recognize that the SST (described in the Appendix), and indeed most cognitive tests, rely on simplistic responses (often finger movements) made by seated participants in response to imperative cues displayed on a computer screen. This study by Rydalch et al. addressed if the ability to stop a prepotent response was preserved across a standard seated test of response inhibition compared with a reactive balance test where compensatory steps must be occasionally suppressed ${ }^{23}$. The results showed a correlation between the cognitive test outcome (stop signal reaction time) and compensatory stepping, which suggests that an individual's stopping capacity generalizes across diverse tasks. 
A
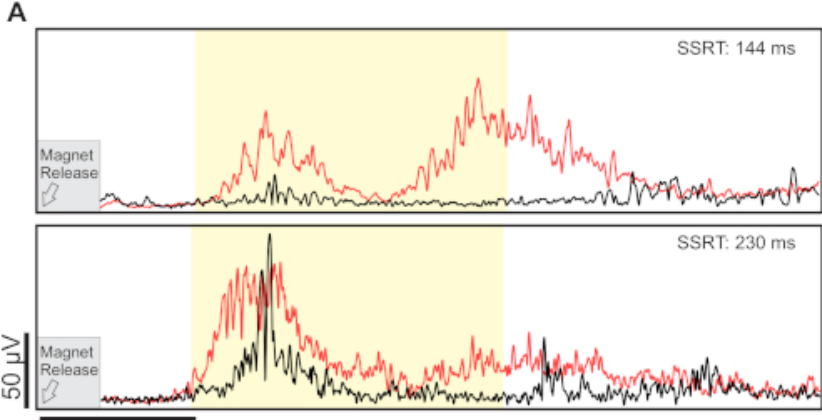

$100 \mathrm{~ms}$

B

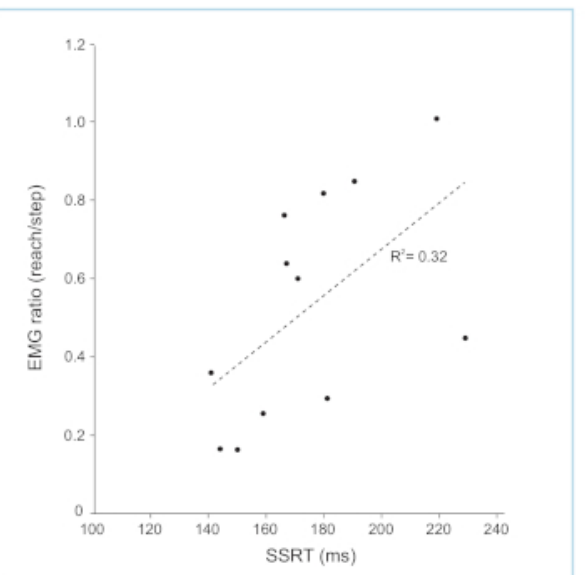

Figure 4. Average step leg response. (A) Average waveforms are shown for the tibialis anterior in the stepping leg. Step trials are shown in red and reach trials in black. Exemplar muscle response data shown for two participants with either a fast (top) or slow stop (bottom) signal reaction time. This stop signal reaction time offers a millisecond measure of stopping ability. The early muscle response (integrated EMG) was measured from 100-300 ms (light yellow shaded region). (B) Scatterplot showing the correlation between the muscle response ratio and stopsignal reaction-time (SSRT) at the $400 \mathrm{~ms}$ visual delay, $r=0.561 ; p=0.029$. Adapted from Figures 3 and 5 , Rydalch et al. ${ }^{23}$. Please click here to view a larger version of this figure.

\section{Example 2}

This study exemplifies how our modified lean \& release setup when combined with TMS can be used to study motor preparation based on vision. The concept of affordances (originally proposed by Gibso ${ }^{24}$ ) was tested in a standing postural context, to determine if corticospinal excitability of a hand muscle (used for grasping) was facilitated when viewing a supportive handle. The key to this approach was assessing how the excitatory state of the motor system was affected by vision alone. Specifically, TMS pulses were delivered shortly after the goggles opened, but prior to any cue for movement (i.e., cable release). In this manner only the motor activity related to the visual scene was analyzed while the behavioral response to perturbation was secondary. Unlike the above study, which emphasized the need for response inhibition by presenting the step response more frequently, this study used an equal probability of handle (reach) vs. no-handle (step) to focus on visual priming of hand action. Results indicated that viewing the handle resulted in facilitation of an intrinsic hand (i.e., grasping) muscle but only in the pure observation condition (Figure 5) ${ }^{21}$. NOTE: For exemplar data, acquisition, and analysis software code, along with guidance notes please refer to the open science framework (https://osf.io/9z3nw/). Examples 1 and 3 used similar code and procedures, with modifications to specific states. 


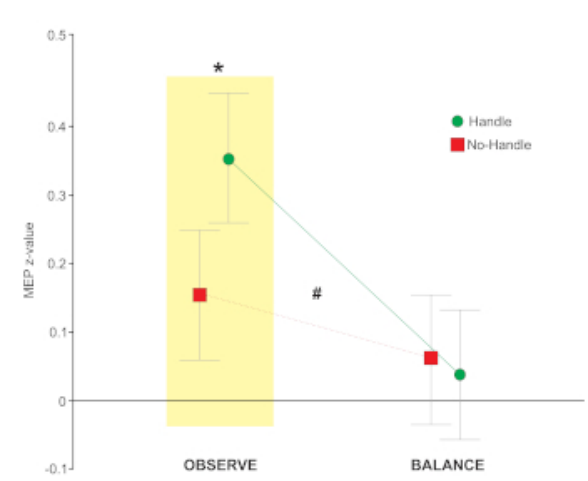

Figure 5. Data showing the difference in corticospinal excitability for the REACH (i.e., handle) versus STEP (i.e., no-handle) trials in an intrinsic hand muscle while participants stood in a supported lean. This showed greater activity in the hand when the handle was present and participants simply viewed the handle (OBS) but this effect was absent during a separate balance (BAL) trials blocks where the cable was periodically released. Error bars show the standard error of the mean. Two-way repeated measures ANOVA revealed an interaction between condition and affordance, $\mathrm{F}_{1,62}=5.69,{ }^{\#} p=0.020$. To address our specific hypotheses, we used prior planned comparisons to determine if MEP amplitude in the FDI was greater when the handle was present within each condition separately. For hypothesis 1 , planned comparisons were used to compare levels of affordance (STEP, REACH) within the OBS condition and revealed a significant increase in amplitude when the handle was visible, $\mathrm{t}_{121}=2.62,{ }^{*} p=0.010$. For hypothesis 2 , we had originally predicted an interaction, but in the opposite direction from what was found. Planned comparison of affordance within the BAL condition showed no significant difference related to the presence of a handle, $\mathrm{t}_{121}=$ $-0.46, p=0.644$. Adapted from Figure 5 , Bolton et al. ${ }^{21}$. Please click here to view a larger version of this figure.

\section{Example 3}

This final example emphasizes how we adapted this device to once again study motor preparation of a hand muscle based on vision but focused on the need to quickly suppress leg action. In this version, the handle cover was permanently covered, while only the leg block moved. Like example 1, the probability of stop versus step conditions was manipulated to encourage an automatic step. Given that the handle was no longer an option in this study, the degree of forward lean measured at the ankle was slightly reduced $\left(\sim 6^{\circ}\right.$ vs. $\sim 10^{\circ}$ as in the above two studies $)$ to allow a fixed support reaction. The specific use for this version of the task was to investigate the concept of global suppression, which has previously been explored in seated tasks where focal button presses were used in response to visual stimuli presented on a computer display ${ }^{25}$. Like example 2, TMS was delivered to assess corticospinal excitability in an intrinsic hand muscle immediately following access to the response environment (i.e., block or no block), but prior to any cue to move (i.e., cable release). The rationale for testing an intrinsic hand muscle in a task that only used leg responses was to see if a task irrelevant muscle would show evidence of a general suppression throughout the motor system. The results depicted below in Figure $\mathbf{6}$ show evidence of a widespread shutdown across the motor system when an automatic step is abruptly stopped $^{22}$. 

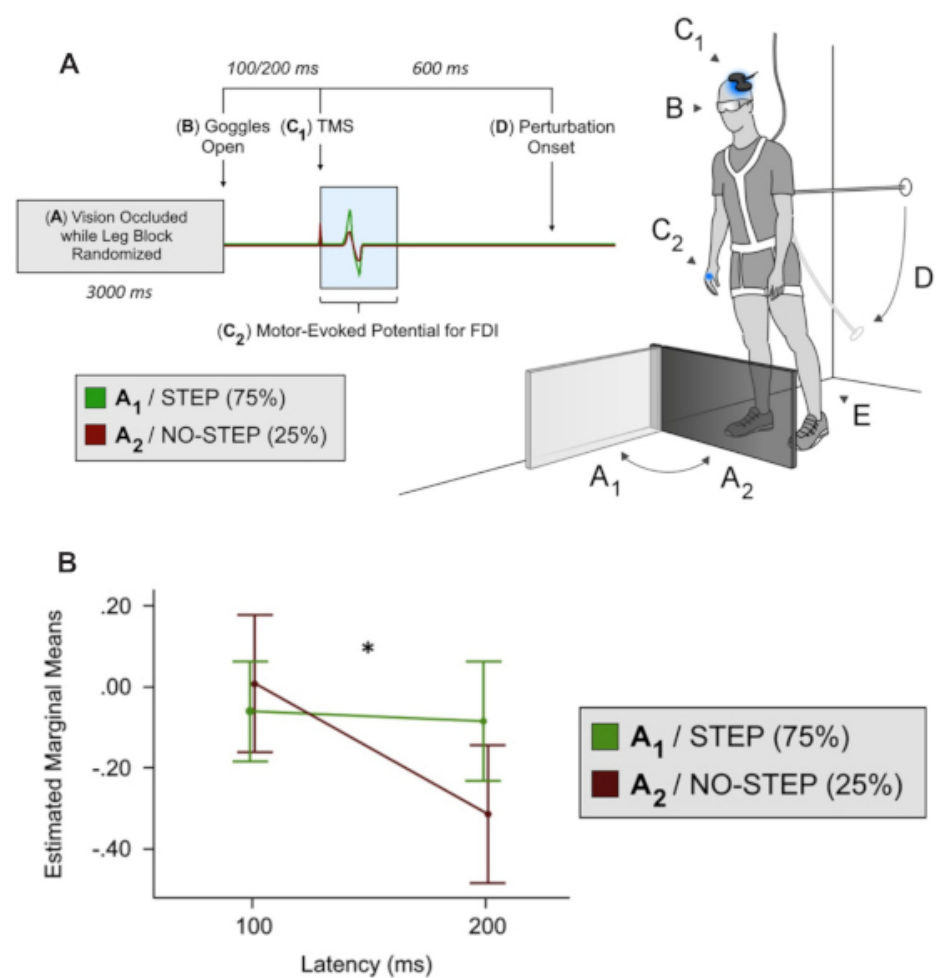

Figure 6. Modified lean \& release task with leg block only (i.e., no option for grasping a support handle). (A) This figure depicts MEP amplitude suppression in an intrinsic hand muscle when a leg block was presented (i.e., NO-STEP condition). (B) From the repeated measures ANOVA, the step condition $\mathrm{x}$ latency interaction, $\mathrm{F}_{1,18}=4.47, p=0.049$, was significant. Visual inspection of the line graph 2 reveals decreasing MEP amplitude over time for the NO-STEP condition only and this was confirmed with follow-up comparisons. Specifically, these comparisons revealed a significant decrease at $200 \mathrm{~ms}$ compared with $100 \mathrm{~ms} \mathrm{t}_{18}=2.595,{ }^{*} p=0.009$ for the NO-STEP condition. By contrast, a similar comparison between $200 \mathrm{~ms}$ and $100 \mathrm{~ms}$ for the STEP condition reveals no difference $\mathrm{t}_{18}=0.346, p=0.367$. Adapted from Figures 1 and 2 , Goode et al. ${ }^{22}$. Please click here to view a larger version of this figure.

\section{Discussion}

This modified lean \& release system provides a novel way to assess cognitive roles in reactive balance. As with the standard lean \& release procedure, the direction and amplitude of postural perturbation are predictable to the subject while the timing of cable release is unpredictable. What is unique in the current approach is that access to vision is precisely controlled while the subject remains fixed and the response environment is altered around them to create different action opportunities and/or constraints. By manipulating the presence of obstacles and affordances this method emphasizes cognitive processes such as decision-making (i.e., action selection) and response inhibition in relation to balance recovery.

The proposed method has potential to provide a unique glimpse into the neural control of balance but poses certain limitations. For example, when using the lean \& release method, the cable release is initiated from a forward lean, which necessitates a pronounced balance recovery step compared with other methods of external postural perturbation ${ }^{10}$. Also, the direction and magnitude of the perturbation are predictable, which may lead to anticipatory activation of muscles that would normally not be engaged in more realistic fall scenarios. Finally, vision is temporarily occluded prior to cable release, which also deviates from an individual's day-to-day experience. These features make our assessment of balance somewhat artificial and may preclude generalization across different modes of perturbation. It is important to recognize that generalizability to real world falls is always a concern when drawing inferences on how balance is controlled from any one particular assessment method. Indeed, a commonly recognized comprehensive test for balance ability does not currently exist ${ }^{4}$. For present purposes, a set forward fall allows perturbation characteristics and response settings to be held constant while manipulating specific cognitive demands that are often neglected or inaccessible in traditional balance assessments. Such experimental control is beneficial but should be taken into consideration when interpreting results.

As a second limitation, the construction of the testing equipment and the requisite engineering skills may represent a challenge to implement this method. Three electrical engineering students from Utah State University built the platform, set up the electronics, and programmed microcontrollers to drive servo-motors for the handle cover and leg block. Construction costs were modest (i.e., $<\$ 15,000$ not including the force plates mounted into the platform). Nevertheless, this may pose a challenge depending on available resources.

Specific insights into the neural control of balance were obtained using this approach. These examples indicate that noninvasive brain stimulation can be used to capture motor set based on viewing objects in a postural context and offer a technique to assess response inhibition using muscle responses. Notably, the modified lean \& release technique could be easily adapted to incorporate other neurophysiological probes such as electroencephalography and functional near-infrared spectroscopy. Even without the inclusion of direct neural measures, study designs that 
focus entirely on external forces, muscle activation, and kinematics can provide important insight into behavioral markers of cognitive deficits. For example, an interesting application for using force plates to capture anticipatory postural shifts during a reactive stepping task has been demonstrated by Cohen et al. ${ }^{26}$. In their study, deficits in response inhibition in older adults were revealed by inappropriate weight shifting, which in turn led to delays in choice-reaction step times. Such an approach could be applied to the current paradigm to gain sensitive measures of weight shifting and stepping errors.

This new method builds from an established reactive balance test where participants are released from a supported lean, and now includes scenarios that demand behavioral flexibility. Test designs suitable for exposing response inhibition and action selection allow us a way to apply concepts from cognitive psychology to the domain of balance control. Such an approach is necessary to build upon the recognition that cognitive decline and fall prevalence are correlated, and to gain a mechanistic understanding for how cognitive resources prevent falls. Presumably this setup could be used not only as a research tool, but also as a means for training cognitive roles in balance. An important aim of ongoing work our laboratory is to understand how the brain utilizes contextual information to update which movement would be most suitable to prevent a fall given the surroundings. Cues such as the availability of a stable handhold or a potential step barrier can guide which response to make should the need arise and may covertly shape predictive brain processes ${ }^{16}$. Notably, the capacity to appropriately use this information may deteriorate with age if mental faculties such as inhibitory interference control or visual-spatial memory are required. Given the relationship between cognitive decline and falls ${ }^{1-3}$, implementing study designs that emphasize a need for integrating contextual relevance could provide valuable insight into balance deficits in many vulnerable populations.

\section{Disclosures}

The authors have nothing to disclose.

\section{Acknowledgments}

Research reported in this publication was supported by the National Institute on Aging of the National Institutes of Health under Award Number R21AG061688. The content is solely the responsibility of the authors and does not necessarily represent the official views of the National Institutes of Health.

\section{References}

1. Mirelman, A. et al. Executive function and falls in older adults: new findings from a five-year prospective study link fall risk to cognition. PloS one. 7 (6), e40297 (2012).

2. Herman, T., Mirelman, A., Giladi, N., Schweiger, A., Hausdorff, J. M. Executive control deficits as a prodrome to falls in healthy older adults: a prospective study linking thinking, walking, and falling. The Journals of Gerontology. Series A, Biological Sciences and Medical Sciences. 65 (10), 1086-1092, (2010).

3. Saverino, A., Waller, D., Rantell, K., Parry, R., Moriarty, A., Playford, E. D. The Role of Cognitive Factors in Predicting Balance and Fall Risk in a Neuro-Rehabilitation Setting. PLOS ONE. 11 (4), e0153469 (2016).

4. Rogers, M. W., Mille, M.-L. Chapter 5 - Balance perturbations. Handbook of Clinical Neurology. 159, $85-105$ (2018).

5. Adkin, A. L., Campbell, A. D., Chua, R., Carpenter, M. G. The influence of postural threat on the cortical response to unpredictable and predictable postural perturbations. Neuroscience Letters. 435 (2), 120-125 (2008).

6. Marlin, A., Mochizuki, G., Staines, W. R., Mcllroy, W. E. Localizing evoked cortical activity associated with balance reactions: does the anterior cingulate play a role? Journal of Neurophysiology. 111 (12), 2634-2643 (2014).

7. Horak, F. B., Nashner, L. M. Central programming of postural movements: adaptation to altered support-surface configurations. Journal of Neurophysiology. 55 (6), 1369-1381 (1986).

8. Nashner, L. M. Fixed patterns of rapid postural responses among leg muscles during stance. Experimental Brain Research. 30 (1), $13-24$ (1977).

9. Varghese, J. P., Marlin, A., Beyer, K. B., Staines, W. R., Mochizuki, G., Mcllroy, W. E. Frequency characteristics of cortical activity associated with perturbations to upright stability. Neuroscience Letters. 578, 33-38 (2014).

10. Mansfield, A., Maki, B. E. Are age-related impairments in change-in-support balance reactions dependent on the method of balance perturbation? Journal of Biomechanics. 42 (8), 1023-1031 (2009).

11. Maki, B. E., Mcllroy, W. E. The role of limb movements in maintaining upright stance: the "change-in-support" strategy. Physical Therapy. $\mathbf{7 7}$ (5), 488-507 (1997)

12. Lakhani, B., Mansfield, A., Inness, E. L., Mcllroy, W. E. Characterizing the determinants of limb preference for compensatory stepping in healthy young adults. Gait \& Posture. 33 (2), 200-204 (2011).

13. Mansfield, A. et al. Training rapid stepping responses in an individual with stroke. Physical Therapy. 91 (6), $958-969$ (2011).

14. Mansfield, A., Inness, E. L., Lakhani, B., Mcllroy, W. E. Determinants of limb preference for initiating compensatory stepping poststroke. Archives of Physical Medicine and Rehabilitation. 93 (7), 1179-1184 (2012).

15. Cheng, K. C., Pratt, J., Maki, B. E. Effects of spatial-memory decay and dual-task interference on perturbation-evoked reach-to-grasp reactions in the absence of online visual feedback. Human Movement Science. 32 (2), 328-342 (2013).

16. Dakin, C. J., Bolton, D. A. E. Forecast or Fall: Prediction's Importance to Postural Control. Frontiers in Neurology. 9, 924 (2018).

17. Slobounov, S., Cao, C., Jaiswal, N., Newell, K. M. Neural basis of postural instability identified by VTC and EEG. Experimental Brain Research. 199 (1), 1-16 (2009).

18. Maki, B. E., Mcllroy, W. E. Cognitive demands and cortical control of human balance-recovery reactions. Journal of Neural Transmission (Vienna, Austria: 1996). 114 (10), 1279-1296 (2007).

19. Bolton, D. A. The role of the cerebral cortex in postural responses to externally induced perturbations. Neuroscience and Biobehavioral Reviews. 57, 142-155 (2015). 
20. Rossi, S., Hallett, M., Rossini, P. M., Pascual-Leone, A., Safety of TMS Consensus Group Safety, ethical considerations, and application guidelines for the use of transcranial magnetic stimulation in clinical practice and research. Clinical Neurophysiology: official journal of the International Federation of Clinical Neurophysiology. 120 (12), 2008-2039, (2009).

21. Bolton, D. A. E. et al. Motor preparation for compensatory reach-to-grasp responses when viewing a wall-mounted safety handle. Cortex. 117, 135-146 (2019).

22. Goode, C., Cole, D. M., Bolton, D. A. E. Staying upright by shutting down? Evidence for global suppression of the motor system when recovering balance. Gait \& Posture. 70, 260-263 (2019).

23. Rydalch, G., Bell, H. B., Ruddy, K. L., Bolton, D. A. E. Stop-signal reaction time correlates with a compensatory balance response. Gait \& Posture. 71, 273-278 (2019).

24. Gibson, J. J. The Ecological Approach To Visual Perception. Houghton Mifflin. Boston. (1979).

25. Majid, D. S. A., Cai, W., George, J. S., Verbruggen, F., Aron, A. R. Transcranial Magnetic Stimulation Reveals Dissociable Mechanisms for Global Versus Selective Corticomotor Suppression Underlying the Stopping of Action. Cerebral Cortex. 22 (2), $363-371$ (2012).

26. Cohen, R. G., Nutt, J. G., Horak, F. B. Errors in postural preparation lead to increased choice reaction times for step initiation in older adults. The Journals of Gerontology. Series A, Biological Sciences and Medical Sciences. 66 (6), 705-713 (2011). 